\title{
THE NONLOCAL PROBLEM FOR THE $2 n$ DIFFERENTIAL EQUATIONS WITH UNBOUNDED OPERATOR COEFFICIENTS AND THE INVOLUTION
}

\begin{abstract}
We study a problem with periodic boundary conditions for a $2 n$-order differential equation whose coefficients are non-self-adjoint operators. It is established that the operator of the problem has two invariant subspaces generated by the involution operator and two subsystems of the system of eigenfunctions which are Riesz bases in each of the subspaces. For a differential-operator equation of even order, we study a problem with non-self-adjoint boundary conditions which are perturbations of periodic conditions. We study cases when the perturbed conditions are Birkhoff regular but not strongly Birkhoff regular or nonregular. We found the eigenvalues and elements of the system $V$ of root functions of the operator which is complete and contains an infinite number of associated functions. Some sufficient conditions for which this system $V$ is a Riesz basis are obtained. Some conditions for the existence and uniqueness of the solution of the problem with homogeneous boundary conditions are obtained.
\end{abstract}

Key words and phrases: operator of involution, differential-operator equation, eigenfunctions, Riesz basis.

${ }^{1}$ Lviv Polytechnic National University, 12 Bandera str., 79013, Lviv, Ukraine

${ }^{2}$ Vasyl Stefanyk Precarpathian National University, 57 Shevchenka str., 76018, Ivano-Frankivsk, Ukraine

E-mail: baryarom@ukr.net (Baranetskij Ya.O.), ihor.demkiv@gmail.com (Demkiv I.I.), vanobsb@gmail.com (Ivasiuk I.Ya.), kopachm2009@gmail.com (Kopach M.I.)

\section{INTRODUCTION}

The theory of differential equations with an unbounded operator coefficient was initiated by Hill and Yosida where the first theorems on the existence of the Cauchy problem solution for a linear homogeneous differential equation with respect to a function with values in a Banach space were obtained. Among works on this subject should be noted works of Kato T. , Krein S.G., Mizohata S., Phillips R.S.

The boundary value problems for linear differential-operator equations are used in the simulation of boundary value problems for differential equations with partial derivatives, in particular, in the study of nonlocal problems. Significant results concerning the theory of boundary value problems for differential-operator equations were obtained in the papers of Vishik M.I., Boehner M., Gorbachuk V.I. and Gorbachuk M.L., Dezin O.O., Dubinsky Yu.V., Kochubei A.N., Lions J.-L., Mamedov K.S., Romanko V.K., Shakhmurov Veli B., Triebel Kh., Yakubov S., Yurchuk N.Yu.

During recent years the number of publications with the use of an involution operator in various sections of the theory of ordinary differential equations (see $[2,8-10,12,13,15,16])$, partial differential equations (see $[1,7,11,14,17,18]$ ) and differential equations with operator coefficients (see [3-6]) increased significantly.

In our article we will use the following notations. Let $H$ be a separable Hilbert space and $A: D(A) \subset H \rightarrow H$ be the closed unbounded linear operator with the discrete spectrum 
$\sigma(A) \equiv\left\{z_{k} \in \mathbb{R}, z_{k}=\alpha(k)^{\gamma}, \alpha, \gamma>0, k=1,2, \ldots\right\}$. We denote by $V(A) \equiv\left\{v_{k} \in H: k=\right.$ $1,2, \ldots\}$ the system of the eigenfunctions of $A$ which forms a Riesz basis in $H$, by $W(A) \equiv$ $\left\{w_{m} \in H: m=1,2, \ldots\right\}$ the biorthogonal system of the functions in the sense of equalities $\left(v_{k}, w_{m} ; H\right)=0, k \neq m,\left(v_{k}, w_{k} ; H\right)=1, k, m=1,2, \ldots ; H\left(A^{s}\right) \equiv\left\{h \in H: A^{s} h \in H\right\}$, $s \geq 0$. Let $H_{1} \equiv L_{2}((0,1), H)$ and $D_{x}: H_{1} \rightarrow H_{1}$ is a strong derivative in the space $H_{1}$; $\left\|\frac{u(x+\triangle x)-u(x)}{\triangle x}-D_{x} u ; H_{1}\right\| \rightarrow 0, \triangle x \rightarrow 0$. Denote by $H_{2} \equiv\left\{u \in H_{1}: D_{x}^{2 n} u \in H_{1}, A^{2 n} u \in H_{1}\right\}$; by $[H]$ the algebra of the bounded linear operators $B: H \rightarrow H$. Denote by $H_{0} \equiv L_{2}(0,1)$; let $I$ be the operator of the involution in the space $H_{0}, I y(x) \equiv y(1-x)$, and let $E$ be the identity transformation in $H_{0}, p_{j} \equiv \frac{1}{2}\left(E+(-1)^{j} I\right)$ are the orthoprojectors in the space $H_{0}$, $H_{0, j} \equiv\left\{y \in H_{0}: y \equiv p_{j} y\right\}, j=0,1$. Let us denote by $W^{2 n}(0,1) \equiv\left\{y \in H_{0}: y^{(m)} \in C[0,1]\right.$, $\left.m=\overline{0,2 n-1}, y^{(2 n)} \in H_{0}\right\}$, by $W^{*}$ the space of the continuous linear functionals on the space $W^{2 n}(0,1)$ and by $W_{j}^{*} \equiv\left\{l \in W^{*}: l y=0, y \in H_{2,1-j} \cap W^{2 n}(0,1)\right\} ; j=0,1$.

We consider the following boundary problem

$$
\begin{aligned}
L w \equiv & (-1)^{n} D_{x}^{2 n} w(x)+A^{2 n} w(x) \\
& +\sum_{s=1}^{n} a_{s}\left(D_{x}^{2 s-1} w(x)+D_{x}^{2 s-1} w(1-x)\right)=f(x), \quad x \in(0,1), \\
\ell_{j} w \equiv & D_{x}^{2 j-1} w(0)-D_{x}^{2 j-1} w(1)+l_{j}^{1} w=\varphi_{j}, \\
\ell_{n+j} w \equiv & D_{x}^{2 j-2} w(0)-D_{x}^{2 j-2} w(1)=\varphi_{n+j},
\end{aligned}
$$

where

$$
\ell_{j}^{1} w \equiv \sum_{r=0}^{m_{j}}\left(b_{j, r, 0} D_{x}^{r} w(0)+b_{j, r, 1} D_{x}^{r} w(1)\right), \quad j=1,2, \ldots, n .
$$

The function $w$ is called the solution of the problem (1)-(4) if

$$
\begin{gathered}
\left\|L w-f ; H_{1}\right\|=0, \quad\left\|l_{j} w-\varphi_{j} ; H\left(A^{\beta_{j}}\right)\right\|=0, \\
\beta_{n+j}=2 n-2 j+\frac{3}{2}, \quad \beta_{j}=2 n-\max \left(m_{j}, 2 j-1\right)-\frac{1}{2}, \\
a_{j}, b_{j, r, s} \in \mathbb{R}, \quad r=0,1, \ldots, \quad m_{j} \leq 2 n-1, \quad s=0,1, \quad j=1,2, \ldots, n .
\end{gathered}
$$

The paper is arranged as follows. In Section 2 we investigate the properties of the operator of problem with periodic conditions for the equation $(-1)^{n} y^{(2 n)}=\lambda y$. In Section 3 we study the spectral properties of the operator of a problem with boundary conditions that are periodic perturbations. In Sections 4 we construct a commutative group of operators that map the root functions of the operators of perturbed boundary-value problems. In Section 5 using these operators, systems of root functions of boundary-value problem operators are constructed and conditions for the completeness and basis property of these systems are established. In Section 6 some analogous results are obtained for the operators of boundary problems generated by differential equations with an involution.

\section{A SPECTRAL PROBLEM WITH PERIODIC BOUNDARY CONDITIONS FOR A DIFFERENTIAL-OPERATOR EQUATION}

Consider the partial case of the problem (1)-(4) with $a_{j}=0, b_{j, r, s}=0, r=0,1, \ldots, m_{j}$, $s=0,1, j=1,2, \ldots, n$, namely

$$
\begin{aligned}
& (-1)^{n} D_{x}^{2 n} u(x)+A^{2 n} u(x)=f(x), \quad x \in(0,1), \\
& \ell_{0, j} u \equiv D_{x}^{2 j-1} u(0)-D_{x}^{2 j-1} u(1)=0, \\
& \ell_{0, n+j} u \equiv D_{x}^{2 j-2} u(0)-D_{x}^{2 j-2} u(1)=0, \quad j=1,2, \ldots, n .
\end{aligned}
$$


Let $L_{0}$ be the operator of the problem (5)-(7),

$$
L_{0} u \equiv(-1)^{n} D_{x}^{2 n} u+A^{2 n} u, \quad u \in D\left(L_{0}\right), D\left(L_{0}\right) \equiv\left\{u \in H_{2}: l_{0, j} u=0, j=1,2, \ldots, 2 n\right\} .
$$

Consider the spectral problem

$$
\begin{aligned}
& (-1)^{n} D_{x}^{2 n} u(x)+A^{2 n} u(x)=\lambda u(x), \quad \lambda \in \mathbb{C}, \\
& \ell_{0, j} u \equiv u^{(2 j-1)}(0)-u^{(2 j-1)}(1)=0, \quad j=1,2, \ldots, 2 n .
\end{aligned}
$$

We find the solution of the spectral problem (8), (9) as the product $u(x)=y(x) v_{k}, v_{k} \in V(A)$, $k=1,2, \ldots$.

To determine the unknown function $y \in W^{2 n}(0,1)$ we obtain the spectral problem

$$
\begin{aligned}
& (-1)^{n} y^{(2 n)}(x)+z_{k}^{2 n} y(x)=\lambda y(x), \quad \lambda \in \mathbb{C}, \\
& \ell_{0, j} y \equiv y^{(2 j-1)}(0)-y^{(2 j-1)}(1)=0, \quad j=1,2, \ldots, n, \\
& \ell_{0, n+j} y \equiv y^{(2 j-2)} y(0)-y^{(2 j-2)}(1)=0, \quad j=1,2, \ldots, n .
\end{aligned}
$$

Let $L_{0, k}$ be the operator of the problem (10)-(12),

$$
L_{0, k} y \equiv(-1)^{n} y^{(2 n)}+z_{k}^{2 n} y, \quad y \in D\left(L_{0, k}\right), D\left(L_{0, k}\right) \equiv\left\{y \in W^{2 n}(0,1): l_{0, j} y=0, j=\overline{1,2 n}\right\} .
$$

The roots $\rho_{j}$ of the characteristic equation $(-1)^{n} \rho^{2 n}=\lambda-z_{k}^{2 n}$ of the differential equation

$$
(-1)^{n} y^{(2 n)}(x)+z_{k}^{2 n} y(x)=\lambda y(x),
$$

are defined by the relations

$$
\rho_{j}=\omega_{j} \rho, \omega_{1}=i, \omega_{j}=i \exp \left(i \frac{\pi(j-1)}{2 n}\right), \quad j=2,3, \ldots, n .
$$

The fundamental system of the solutions of the differential equation (13) is defined by the formulas

$$
\begin{aligned}
& Y_{j}(x, \rho) \equiv \frac{1}{2}\left(\exp \omega_{j} \rho x+\exp \omega_{j} \rho(1-x)\right) \in H_{2,0}, \quad j=1,2, \ldots, n, \\
& Y_{n+j}(x, \rho) \equiv \frac{1}{2}\left(\exp \omega_{j} \rho x-\exp \omega_{j} \rho(1-x)\right) \in H_{2,1}, \quad j=1,2, \ldots, n .
\end{aligned}
$$

Substituting the general solution

$$
y(x, \rho)=\sum_{s=1}^{2 n} C_{s} Y_{s}(x, \rho)
$$

of the differential equation (13) into the boundary conditions (11), (12) we obtain the equation for determination the eigenvalues of the operator $L_{0, k}$

$$
\Delta(\rho)=\operatorname{det}\left(l_{r} Y_{j}\right)_{r, j=1}^{2 n}=0 .
$$

By substituting the functions (14), (15) in the boundary conditions (11), (12), we obtain $l_{0, r} Y_{n+j}=0, l_{0, n+r} Y_{j}=0, j, r=1,2, \ldots, n$. Therefore $\Delta(\rho)=\Delta_{0}(\rho) \Delta_{1}(\rho)=0$, where $\Delta_{s}(\rho)=\operatorname{det}\left(l_{s n+r} Y_{s n+j}\right)_{r, j=1}^{n}, s=0,1$.

The operator $L_{0, k}$ is self-adjoint (see [15]). Therefore the solutions of the equation (16) are $\rho_{q}=2 q \pi i, q=0,1,2, \ldots$, which are numbered in ascending order and lie on the half-line $\operatorname{Im} \rho=0, \operatorname{Re} \rho \geq 0$.

Thus, the operator $L_{0, k}$ has eigenvalues $\lambda_{q, k}=\left(\rho_{q}\right)^{2 n}+z_{k}^{2 n}, q=0,1, \ldots$ We obtain the following result. 
Lemma 2.1. The self-adjoint operator $L_{0, k}$ has a point spectrum

$$
\sigma\left(L_{0, k}\right)=\left\{\lambda_{q} \in \mathbb{R}: \lambda_{q}=(2 \pi q)^{2 n}+z_{k}^{2 n}, q=0,1, \ldots\right\}
$$

and a system of eigenfunctions

$$
\begin{gathered}
V\left(L_{0, k}\right) \equiv\left\{v_{q}(x) \in L_{2}(0,1): v_{0}(x)=1, v_{2 q}(x) \equiv \sqrt{2} \cos 2 \pi q x,\right. \\
\left.v_{2 q-1}(x) \equiv \sqrt{2} \sin 2 \pi q x, q=1,2, \ldots\right\}
\end{gathered}
$$

which is an orthonormal basis of the space $H_{0}$.

Remark 2.1. The systems

$$
V_{0}\left(L_{0, k}\right) \equiv\left\{v_{2 q}(x): q=0,1, \ldots\right\}, V_{1}\left(L_{0, k}\right) \equiv\left\{v_{2 q-1}(x): q=1,2, \ldots\right\}
$$

form an orthonormal basis in spaces $H_{0,0}$ and $H_{0,1}$, respectively.

Therefore, the operator $L_{0}$ has the following eigenfunctions in the space $H_{1}$

$$
V\left(L_{0}\right) \equiv\left\{v_{q, k}\left(x, L_{0}\right) \in H_{1}: v_{q, k}\left(x, L_{0}\right) \equiv v_{q}(x) v_{k}, q=\overline{0, \infty}, k=\overline{1, \infty}\right\} .
$$

A system of functions $\left\{h_{s}\right\}_{s=1}^{\infty} \subset H$ is called a Riesz basis in a Hilbert space $H$, if $\left\{h_{s}\right\}_{s=1}^{\infty}$ is complete in the space $H$, and for any orthonormal basis $\left\{e_{s}\right\}_{s=1}^{\infty} \subset H$ there exists an isomorphism $B: H \rightarrow H, B e_{s}=h_{s}, s=1,2, \ldots$.

The product of a system $V(A)$ and an orthonormal system $V\left(L_{0, k}\right)$ is the Riesz basis (see [9]) in the space $H_{1}$. Thus, the following theorem is true.

Theorem 1. The operator $L_{0}$ has a discrete spectrum

$$
\sigma\left(L_{0}\right) \equiv\left\{\lambda_{q, k} \in \mathbb{R}: \lambda_{q, k} \equiv \rho_{q}^{2 n}+z_{k}^{2 n}, k=\overline{1, \infty}, q=\overline{0, \infty}\right\},
$$

and the system of the eigenfunctions $V\left(L_{0}\right)$ forms the Riesz basis in the space $H_{1}$.

Let us consider the functions

$$
\begin{aligned}
& y_{r}\left(x, \rho_{q}\right) \equiv \frac{1}{2}\left(1+e^{\omega_{r} \rho_{q}}\right)^{-1}\left(e^{\omega_{r} \rho_{q} x}+e^{\omega_{r} \rho_{q}(1-x)}\right), \\
& y_{1}\left(x, \rho_{q}\right) \equiv \frac{1}{2}(1-2 x) \sin \rho_{q} x, \quad r=2,3, \ldots, n, q=1,2, \ldots,
\end{aligned}
$$

and determine the square matrix

$$
\mathrm{B}_{0, p}\left(x, \rho_{q}\right) \equiv\left(\beta_{p, s}^{0}\right)_{p, s=1}^{n}
$$

of the order $n$ according to the following: the row with number $p$ is determined by the elements of the system (17) $\beta_{p, s}^{0}\left(x, \rho_{q}\right) \equiv y_{s}\left(x, \rho_{q}\right)$ and the other lines by the formulas $\beta_{j, s}^{0}\left(x, \rho_{q}\right) \equiv$ $\left(\omega_{s}\right)^{2 j-1}$ with $j \neq p, j, s=1,2, \ldots, n$.

We denote the determinant of the matrix $\mathrm{B}_{0, p}\left(x, \rho_{q}\right)$ by $y_{1, p}\left(x, \rho_{q}\right)$.

Substituting the determinant into conditions (11), (12), we obtain

$$
l_{0, r} y_{1, p}=0, r \neq p, l_{0, p} y_{1, p}=\left(\rho_{q}\right)^{2 p-1} h(i) W^{n},
$$


where the Vandermonde determinant $W^{n}$ is constructed by the numbers

$$
1,\left(\omega_{2}\right)^{2}, \ldots,\left(\omega_{n}\right)^{2}, h(i)=(-i)^{n-1} i, i=\sqrt{-1}, r=1, \ldots, 2 n, m=1,2, \ldots, n .
$$

Consider the functions $y_{2, p}\left(x, \rho_{q}\right) \equiv\left(h(i) W^{n}\right)^{-1} y_{1, p}\left(x, \rho_{q}\right), p=1,2, \ldots, n$.

From the relation (18) we obtain

$$
l_{0, r} y_{2, p}=0, r \neq p, l_{0, p} y_{2, p}=\left(\rho_{q}\right)^{2 p-1}, p, r=1,2, \ldots, n \text {. }
$$

Similarly, let us consider the system of functions

$$
\begin{aligned}
& y_{n+r}\left(x, \rho_{q}\right) \equiv \frac{1}{2}\left(1-e^{w_{r} \rho_{q}}\right)^{-1}\left(e^{w_{r} \rho_{q} x}-e^{w_{r} \rho_{q}(1-x)}\right), \\
& y_{n+1}\left(x, \rho_{q}\right) \equiv \frac{1}{2}(1-2 x) \cos \rho_{q} x, \quad r=2,3, \ldots, n, q=1,2, \ldots,
\end{aligned}
$$

and a square matrix

$$
\mathrm{B}_{1, r}\left(x, \rho_{q}\right) \equiv\left(\beta_{p, s}^{1}\right)_{p, s=1}^{n}
$$

of the order $n$ which rows are determine by following: the row with number $r$ is determined by the elements of the system $(20) \beta_{r, s}^{1}\left(x, \rho_{q}\right) \equiv y_{n+s}\left(x, \rho_{q}\right)$ and the other lines by the equalities

$$
\beta_{j, s}^{1}\left(x, \rho_{q}\right) \equiv\left(\omega_{s}\right)^{2 j-2}, j \neq r, r, s=1,3, \ldots, n .
$$

We denote the determinant of the matrix by $y_{1, n+r}\left(x, \rho_{q}\right)$.

Substituting it into conditions (11), (12), we get

$$
l_{0, j} y_{1, n+r}=0, j \neq n+r, l_{0, n+r} y_{1, n+r}=W^{n}\left(\rho_{q}\right)^{2 r-2}
$$

Let us define the functions $y_{2, n+r}\left(x, \rho_{q}\right) \equiv\left(W^{n}\right)^{-1} y_{1, n+r}\left(x, \rho_{q}\right), r=1,2, \ldots, n$.

Taking the relation (21) for the functions $y_{2, n+r}\left(x, p_{q}\right)$ into account, we obtain

$$
l_{0, j} y_{2, n+r}=0, j \neq n+r, l_{0, n+r} y_{2, n+r}=\left(\rho_{q}\right)^{2 r-2}, j=1, \ldots, 2 n, r=1,2, \ldots, n \text {. }
$$

Remark 2.2. There exist positive numbers $K_{0}, K_{1}$ such that

$$
K_{1} \leq\left\|y_{2, j}\left(x, \rho_{q}\right) ; H_{0}\right\| \leq K_{2}<\infty, \quad j=1,2, \ldots, 2 n, q=1,2, \ldots .
$$

Here $K_{s}, s \in \mathbb{N}$, are positive constants.

\section{NONLOCAL BOUNDARY VALUE PROBLEM}

For the differential-operator equation (5) and an arbitrary fixed $p \in\{1,2, \ldots, n\}$ and $b \in \mathbb{R}$ we consider the boundary value problem

$$
\begin{aligned}
& \ell_{1, j} u \equiv D_{x}^{2 j-1} u(0)-D_{x}^{2 j-1} u(1)=0, \quad j \neq p, j=1,2, \ldots, n, \\
& \ell_{1, p} u \equiv D_{x}^{2 p-1} u(0)-D_{x}^{2 p-1} u(1)+l_{p}^{2} u=0, \\
& \ell_{1, n+j} u \equiv D_{x}^{2 j-2} u(0)-D_{x}^{2 j-2} u(1)=0, \quad j=1,2, \ldots, n,
\end{aligned}
$$

with

$$
\ell_{p}^{2} u \equiv b\left(D_{x}^{2 p-1} u(0)+D_{x}^{2 p-1} u(1)\right)=0, \quad b \in \mathbb{R} .
$$


We will use following notations. Let $L_{1}$ be the operator of the problem (5), (23)-(26) and $L_{1} u \equiv$ $(-1)^{n} D_{x}^{2 n} u(x)+A^{2 n} u(x), u \in D\left(L_{1}\right), D\left(L_{1}\right) \equiv\left\{u \in H_{2}: l_{1, r} u=0, r=\overline{1,2 n}\right\}$.

We find the solution of the spectral problem (8), (23)-(26) as the product $u(x)=y(x) v_{k}$, $v_{k} \in V(A), k=1,2, \ldots$

To determine the unknown function $y \in W^{2 n}(0,1)$ we consider the spectral problem

$$
\begin{aligned}
& (-1)^{n} y^{(2 n)}(x)+z_{k}^{2 n} y(x)=\lambda y(x), \quad \lambda \in \mathbb{C}, \\
& \ell_{1, j} y \equiv y^{(2 j-1)}(0)-y^{(2 j-1)}(1)=0, \quad j \neq p, j=1,2, \ldots, n, \\
& \ell_{1, p} y \equiv y^{(2 p-1)}(0)-y^{(2 p-1)}(1)+l_{p}^{2} y=0, \\
& l_{1, n+j} y \equiv y^{(2 j-2)}(0)-y^{(2 j-2)}(1)=0, \quad j=1,2, \ldots, n,
\end{aligned}
$$

with

$$
l_{p}^{2} y \equiv b\left(y^{(2 p-1)}(0)+y^{(2 p-1)}(1)\right) .
$$

Let $L_{1, k} \equiv L_{1, k, p}$ be the operator of the problem (27)-(31) and

$L_{1, k} y \equiv(-1)^{n} y^{(2 n)}(x)+z_{k}^{2 n} y(x), \quad y \in D\left(L_{1, k}\right), D\left(L_{1, k}\right) \equiv\left\{y \in W^{2 n}(0,1): l_{1, j} y=0, j=\overline{1,2 n}\right\}$.

Let $V\left(L_{1, k}\right)$ be the system of root functions of the operator $L_{1, k}$, let $R\left(L_{1, k}\right) \equiv E+S\left(L_{1, k}\right)$ be the operator which maps the system $V\left(L_{0, k}\right)$ into the system $V\left(L_{1, k}\right)$.

Theorem 2. For any $b \in \mathbb{R}, p \in\{1,2, \ldots, n\}$, the operator $L_{1, k}$ has the point spectrum $\sigma\left(L_{0, k}\right)$ and the system of root functions $V\left(L_{1, k}\right)$ forms a Riesz basis in $H_{0}$.

Proof. We will show that eigenvalues of the operators $L_{0, k}$ and $L_{1, k}$ coincide.

We substitute the fundamental system (14), (15) for the solutions of the differential equation (27) into the boundary conditions (28)-(31). Using $l_{p}^{2} y_{n+j}(x, \rho)=0, j, p=\overline{1, n}$, we obtain the same equations for determination the spectrum

$$
\operatorname{det}\left(l_{1, j} y_{r}(x, \rho)\right)_{j, r=1}^{2 n}=\operatorname{det}\left(l_{1, j} y_{r}(x, \rho)\right)_{j, r=1}^{n} \operatorname{det}\left(l_{1, n+j} y_{n+r}(x, \rho)\right)_{j, r=1}^{n} .
$$

Let us define elements of the system $V\left(L_{1, k}\right)$.

It is easy to see that $v_{2 q}(x) \in D\left(L_{1, k}\right), L_{1, k} v_{2 q}(x)=\lambda_{q} v_{2 q}(x), q=0,1,2, \ldots$. Hence

$$
v_{2 q}\left(x, L_{1, k}\right) \equiv v_{2 q}(x), \quad q=0,1, \ldots .
$$

We define the root functions of the operator $L_{1, k}$ as

$$
v_{2 q-1}\left(x, L_{1, k}\right) \equiv v_{2 q-1}(x)+c_{b, p} y_{2, p}\left(x, \rho_{q}\right) .
$$

Substituting (33) into the boundary condition (29) and taking the equality (19) into account, we obtain $c_{b, p}=-\sqrt{2} b$,

$$
v_{2 q-1}\left(x, L_{1, k}\right) \equiv v_{2 q-1}(x)-\sqrt{2} b y_{2, p}\left(x, \rho_{q}\right), \quad q=1,2, \ldots
$$

Thus, the operator $L_{1, k}$ has a system of root functions (32)-(34) in the sense of equalities

$$
\begin{aligned}
& L_{1, k} v_{2 q-1}\left(x, L_{1, k}\right)=\lambda_{q, k} v_{2 q-1}\left(x, L_{1, k}\right)+\xi_{b, q} v_{2 q}\left(x, L_{1, k}\right), \\
& \xi_{b, q}=-4 \sqrt{2} b n\left(\rho_{q}\right)^{2 n-1}, L_{1, k} v_{2 q}\left(x, L_{1, k}\right)=\lambda_{q, k} v_{2 q}\left(x, L_{1, k}\right), \quad q=1,2, \ldots
\end{aligned}
$$

Given the regularity according to Birkhoff (see [15]) of boundary conditions (28)-(31) we obtain that the system $V\left(L_{1, k}\right)$ is complete and minimal in the space $H_{0}$. 
Let $W^{n-1}$ be the Vandermonde determinant constructed by elements $\omega_{2}^{2}, \omega_{3}^{2}, \ldots, \omega_{n}^{2}$; let $R_{1} \equiv E+S_{1}$ be the operator which maps the system $V\left(L_{0}\right)$ into the system $V_{1}$ and elements of this system are

$$
\begin{aligned}
& v_{2 q-1,1}(x) \equiv\left(1-\sqrt{2}^{-1} W^{n}\left(h(i) W^{n-1}\right)^{-1} b(1-2 x)\right) v_{2 q-1}(x), \\
& v_{0,1}(x) \equiv v_{0}(x), v_{2 q, 1}(x) \equiv v_{2 q}(x), \quad q=1,2, \ldots
\end{aligned}
$$

Lemma 3.1. The system $V_{1}$ forms a Riesz basis in $H_{0}$.

Proof. For an arbitrary function $\varphi \in H_{0}$ we have

$$
\begin{aligned}
& \varphi=\varphi_{0} v_{0}(x)+\sum_{q=0}^{\infty}\left(\varphi_{2 q} v_{2 q}(x)+\varphi_{2 q-1} v_{2 q-1}(x)\right) \in H_{0} \\
& \left\|\varphi ; H_{0}\right\|^{2}=\left|\varphi_{0}\right|^{2}+\sum_{q=1}^{\infty}\left(\left|\varphi_{2 q}\right|^{2}+\left|\varphi_{2 q-1}\right|^{2}\right)<\infty
\end{aligned}
$$

consider the function

$$
\begin{aligned}
& \varphi_{1}=R_{1} \varphi=\varphi_{0} v_{0,1}(x)+\sum_{q=1}^{\infty}\left(\varphi_{2 q} v_{2 q, 1}(x)+\varphi_{2 q-1} v_{2 q-1,1}(x)\right), \\
& \left\|R_{1} \varphi ; H_{0}\right\|^{2} \leq K_{3}\left\|\varphi ; H_{0}\right\|^{2}, \quad K_{3}=2\left(1+\left|W^{n}\left(W^{n-1}\right)^{-1} b\right|^{2}\right) .
\end{aligned}
$$

Therefore $\left\|R_{1} ;\left[H_{0}\right]\right\|^{2} \leq K_{3}<\infty, R_{1} \equiv E+S_{1} \in\left[H_{0}\right], R_{1}^{-1} \equiv E-S_{1} \in\left[H_{0}\right]$. Taking into account the Bari Theorem (see [9]), we obtain the following statement: the system $V_{1}$ forms the Riesz basis in $H_{0}$.

Therefore, the operator $L_{1}$ has the following system of root functions in the space $H_{1}$

$$
V\left(L_{1}\right) \equiv\left\{v_{q, k}\left(x, L_{1}\right) \equiv v_{q}\left(x, L_{1, k}\right) v_{k} \in H_{1}: q=\overline{0, \infty}, k=\overline{1, \infty}\right\} .
$$

Remark 3.1. The operator $L_{1}$ has a system of root functions in the means of equalities

$$
\begin{aligned}
& L_{1} v_{2 q-1, k}\left(x, L_{1}\right)=\lambda_{q, k} v_{2 q-1, k}\left(x, L_{1}\right)+\xi_{b, q} v_{2 q, k}\left(x, L_{1}\right), \\
& \xi_{b, q}=-4 \sqrt{2} b n\left(\rho_{q}\right)^{2 n-1}, \quad q, k=1,2, \ldots, \\
& L_{1} v_{2 q, k}\left(x, L_{1}\right)=\lambda_{q, k} v_{2 q, k}\left(x, L_{1}\right), \quad q=0,1, \ldots, k=1,2, \ldots
\end{aligned}
$$

Theorem 3. For any fixed numbers $p \in\{1,2, \ldots, n\}, b \in \mathbb{R}$, the system $V\left(L_{1}\right)$ is the Riesz basis of the space $H_{1}$.

Proof. Let $R\left(L_{1, k}\right) \equiv E+S\left(L_{1, k}\right): V\left(L_{0, k}\right) \rightarrow V\left(L_{1, k}\right)$, let $p_{k}$ be a projection in $H, p_{k} y \equiv$ $\left(y, w_{k}(A) ; H\right) v_{k}, R\left(L_{1}\right) \equiv \sum_{k=1}^{\infty} R\left(L_{1, k}\right) p_{k}$.

From the definition of the operator $R\left(L_{1}\right)=E+S\left(L_{1}\right)$ it follows that $R^{-1}\left(L_{1}\right)=E-S\left(L_{1}\right)$.

Therefore the system $V\left(L_{1}\right)$ is complete and minimal in the space $H_{1}$. Taking into account the representations of the elements of the system $V\left(L_{1, k}\right)$ and Theorem 2, we obtain $\left\|R\left(L_{1}\right) ;\left[H_{1}\right]\right\| \leq K_{4}\left\|R\left(L_{1, k}\right) ;\left[H_{0}\right]\right\|<\infty$.

Taking into account the Bari Theorem (see [9]), we obtain the following statement: the system $V_{1}$ forms the Riesz basis in $H_{1}$. 


\section{TRANSFORMATION OPERATORS}

For any $k \in \mathbb{N}, p \in\{1,2, \ldots, n\}$, we define the operator $B_{p}: H_{0} \rightarrow H_{0}$ as the operator whose eigenvalues coincide with eigenvalues of the operator $L_{0, k}$, and the root functions are defined by

$$
v_{2 s}\left(x, B_{p}\right) \equiv v_{2 s}(x), v_{2 q-1}\left(x, B_{p}\right) \equiv v_{2 q-1}(x)+c_{q}\left(B_{p}\right) y_{2, p}\left(x, \rho_{q}\right),
$$

where $c_{q}\left(B_{p}\right) \in \mathbb{R}, s=0,1, \ldots, q=1,2, \ldots$.

The operator which maps the system $V\left(L_{0, k}\right)$ into the system $V\left(B_{p}\right)$ of the root functions of the operator $B_{p}$ is denoted by $R\left(B_{p}\right) \equiv E+S\left(B_{p}\right)$, where $S\left(B_{p}\right): H_{0,0} \rightarrow H_{0,1}, S\left(B_{p}\right): H_{0,1} \rightarrow 0$.

We denote by $G_{0, p}\left(L_{0, k}\right) \equiv\left\{R\left(B_{p}\right)\right\}$ such that the root functions of the operator $B_{p}$ are defined by the equalities (36), and $G_{0, p, c}\left(L_{0, k}\right) \equiv G_{0, p}\left(L_{0, k}\right) \cap\left[H_{0}\right]$.

Remark 4.1. Using that $S\left(B_{p}\right): H_{0,0} \rightarrow H_{0,1}, S\left(B_{p}\right): H_{0,1} \rightarrow 0$ we obtain $S^{2}\left(B_{p}\right)=0$, $R^{-1}\left(B_{p}\right) \equiv E-S\left(B_{p}\right)$.

Consequently, the operator $R\left(B_{p}\right)$ has a dense domain in the space $H_{0}$ and the system of root functions is complete and minimal in $H_{0}$.

Similarly, using the root functions of an adjoint operator $L_{1, k}^{*}$, we define the functions

$$
\begin{aligned}
& w_{0}\left(x, B_{p}\right) \equiv v_{0}(x)+c_{0}(1-2 x), \\
& w_{2 q}\left(x, B_{p}\right) \equiv v_{2 q}(x)+c_{q}\left(B_{p}\right) y_{2,2 n-p-1}\left(x, \rho_{q}\right), w_{2 q-1}\left(x, B_{p}\right) \equiv v_{2 q-1}(x), \quad q=1,2, \ldots,
\end{aligned}
$$

and the set of operators $G_{1, p}\left(L_{0, k}\right) \equiv\left\{R\left(B_{p}^{*}\right)=E+S\left(B_{p}^{*}\right), R\left(B_{p}\right) \in G_{0, p}\left(L_{0, k}\right)\right\}$.

Theorem 4. For any $b \in \mathbb{R}, p \in\{1,2, \ldots, n\}$, the operator $B_{p}$ has the point spectrum $\sigma\left(L_{0, k}\right)$ and the system of root functions $V\left(L_{1, k}\right)$ forms the Riesz basis in $H_{0}$ if and only if the sequence $c_{q}\left(B_{p}\right)$ is bounded, i.e. $\left|c_{q}\left(B_{p}\right)\right| \leq K_{5}<\infty, q=1,2, \ldots$

Proof. The necessity. Let the system $V\left(B_{p}\right)$ be the Riesz basis in $H_{0}$, i.e. $R\left(B_{p}\right) \in\left[H_{0}\right]$, then $S\left(B_{p}\right)=E-R_{p}(B) \in\left[H_{0}\right]$. From the definition of the operator $B_{p}$ we have

$$
S\left(B_{p}\right) v_{2 q-1}(x)=c_{q}\left(B_{p}\right) y_{2, p}\left(x, \rho_{q}\right), \quad q=1,2, \ldots
$$

Therefore, taking the estimate (22) into account, we obtain

$$
\begin{aligned}
& \left|c_{q}\left(B_{p}\right)\right| \leq\left\|S\left(B_{p}\right) ;\left[H_{0}\right]\right\|\left\|y_{2, p}\left(x, \rho_{q}\right) ; H_{0}\right\|^{-1} \leq K_{6}<\infty, \\
& K_{6}=K_{5}^{-1}\left\|S\left(B_{p}\right) ;\left[H_{0}\right]\right\|, \quad q=1,2, \ldots
\end{aligned}
$$

The sufficiency. The completeness of the system $V\left(B_{p}\right)$ in the space $H_{0}$ follows from Remark 4.1.

Let $\varphi \in H_{0}, \varphi=\varphi_{0}+\varphi_{1}, \varphi_{s} \in H_{0, s}, s=0,1$. Then we have

$$
\begin{aligned}
& \varphi=\varphi_{0} v_{0}(x)+\sum_{q=1}^{\infty}\left(\varphi_{2 q} v_{2 q}(x)+\varphi_{2 q-1} v_{2 q-1}(x)\right) \in H_{0}, \\
& \left\|\varphi ; H_{0}\right\|^{2}=\left|\varphi_{0}\right|^{2}+\sum_{q=1}^{\infty}\left(\left|\varphi_{2 q}\right|^{2}+\left|\varphi_{2 q-1}\right|^{2}\right)<\infty \\
& R\left(B_{p}\right) \varphi=\varphi_{0} v_{0}\left(x, B_{p}\right)+\sum_{q=1}^{\infty}\left(\varphi_{2 q} v_{2 q}\left(x, B_{p}\right)+\varphi_{2 q-1} v_{2 q-1}\left(x, B_{p}\right)\right) \in H_{0}, \\
& R\left(B_{p}\right) \varphi=\varphi_{0} v_{0}\left(x, B_{p}\right)+\sum_{q=1}^{\infty}\left(\varphi_{2 q} v_{2 q}(x)+\varphi_{2 q-1} c_{q}\left(B_{p}\right)\left(v_{2 q-1}\left(x, L_{1, k}\right)-v_{2 q-1}(x)\right)\right), \\
& \left\|R\left(B_{p}\right) \varphi ; H_{0}\right\|^{2} \leq K_{7}\left\|\varphi ; H_{0}\right\|^{2}, K_{7}=3\left(1+K_{6}^{2}+K_{6}^{2}\left\|R\left(L_{1, k}\right) ;\left[H_{0}\right]\right\|^{2}\right) .
\end{aligned}
$$


Therefore, $\left\|R\left(B_{p}\right) ;\left[H_{0}\right]\right\|^{2} \leq K_{7}<\infty$.

Consider equalities $R\left(B_{p}\right)=E+S\left(B_{p}\right), R^{-1}\left(B_{p}\right)=E-S\left(B_{p}\right)$. We have

$$
R^{-1}\left(B_{p}\right)=2 E-R\left(B_{p}\right) .
$$

Therefore, $\left\|R^{-1}\left(B_{p}\right) ;\left[H_{0}\right]\right\|^{2} \leq K_{8}<\infty, K_{8}=8+2 K_{7}$. Taking into account the Bari Theorem (see [9]), we obtain that the system $V_{1}$ forms the Riesz basis in $H_{0}$.

Suppose that $Q_{0}(I)$ is a set of operators $R=E+S$, such that $S: H_{0,0} \rightarrow H_{0,1}, S: H_{0,1} \rightarrow 0$, $Q_{0, c}(I) \equiv\left[H_{0}\right] \cap Q_{0}(I)$. Using that $S^{2}\left(B_{p}\right)=0, R\left(B_{p}\right) \in G_{0, p}\left(L_{0, k}\right) \subset Q_{0}(I)$ on the set $Q_{0}(I)$, we can define the operation of multiplication

$$
R_{1} R_{2} \equiv\left(E+S_{1}\right)\left(E+S_{2}\right)=E+S_{1}+S_{2}, R_{1}, R_{2} \in Q_{0}(I) .
$$

In particular, $(E+S)(E-S)=E-S^{2}=E, R=E+S \in Q_{0}(I)$.

Therefore, for each operator $R=E+S \in Q_{0}(I)$ there exists a unique inverse operator $R^{-1}=E-S$.

According to the definition of the operator $B_{p}$ and the set $G_{0, p}\left(L_{0, k}\right)$ we have the inclusions

$$
G_{0, p}\left(L_{0, k}\right) \subset Q_{0}(I), G_{c, 0, p}\left(L_{0, k}\right) \subset Q_{0, c}(I), \quad p \in\{1,2, \ldots, n\} .
$$

Thus, the set $Q_{0}(I)$ is an Abelian group which contains the Abelian subgroups $Q_{c, 0}(I)$, $G_{0, p}\left(L_{0, k}\right), G_{0, c, p}\left(L_{0, k}\right), p \in\{1,2, \ldots, n\}$. Therefore, for all operators $R_{j}=E+S_{j} \in Q_{0}(I)$, $j=1,2 \ldots, d, d \in \mathbb{N}$, the following equality

$$
\prod_{j=1}^{d} R_{j} \equiv \prod_{j=1}^{d}\left(E+S_{j}\right)=E+\sum_{j=1}^{d} S_{j}, \quad d \in \mathbb{N},
$$

holds.

\section{NONLOCAL BOUNDARY VALUE PROBLEMS FOR A DIFFERENTIAL-OPERATOR EQUATION}

5.1. For the differential-operator equation (5) and arbitrary fixed indices $b_{p, r, s} \in \mathbb{R}, p \in$ $\{1,2, \ldots, n\}, r=0,1, \ldots, k_{j}, s=0,1, j=1,2, \ldots, n$, we consider the boundary problem generated by nonlocal conditions

$$
\begin{aligned}
& \ell_{2, j} w \equiv D_{x}^{2 j-1} w(0)-D_{x}^{2 j-1} w(1)=0, \quad j \neq p, \\
& \ell_{2, p} w \equiv D_{x}^{2 p-1} w(0)-D_{x}^{2 p-1} w(1)+l_{p}^{1} w=0, \\
& \ell_{2, n+j} w \equiv D_{x}^{2 j-2} w(0)-D_{x}^{2 j-2} w(1)=0, \quad j=1,2, \ldots, n,
\end{aligned}
$$

where

$$
\ell_{p}^{1} w \equiv \sum_{r=0}^{m_{p}}\left(b_{p, r, 0} D_{x}^{r} w(0)+b_{j, r, 1} D_{x}^{r} w(1)\right)
$$

Assumption $P_{1}: b_{p, r, 0}=(-1)^{r+1} b_{p, r, 1}, r=0,1, \ldots, m_{p}, j, p=1,2, \ldots, n$.

Assumption $P_{2}$ : $m_{p} \leq 2 p-1, p=1,2, \ldots, n$.

Remark 5.1. Assumption $P_{1}$ implies that $l_{p}^{1} \in W_{1}^{*}, p=1,2, \ldots, n$. 
Let $L_{2} \equiv L_{2, p}$ be the operator of the problem (5), (38)-(41) and

$L_{2} u \equiv(-1)^{n} D_{x}^{2 n} u(x)+A^{2 n} u(x), \quad u \in D\left(L_{2}\right), D\left(L_{2}\right) \equiv\left\{u \in H_{2}: l_{2, j} u=0, j=1,2, \ldots, 2 n\right\}$.

The solution of the spectral problem (5), (38)-(41) is defined as the product $w(x)=y(x) v_{k}$, $v_{k} \in V(A), k=1,2, \ldots$

To determine the unknown function $y \in W^{2 n}(0,1)$ we consider the spectral problem

$$
\begin{aligned}
& (-1)^{n} y^{(2 n)}(x)+z_{k}^{2 n} y(x)=\lambda y(x), \quad \lambda \in \mathbb{C}, \\
& \ell_{2, j} y \equiv y^{(2 j-1)}(0)-y^{(2 j-1)}(1)=0, \quad j \neq p, \\
& \ell_{2, p} y \equiv y^{(2 p-1)}(0)-y^{(2 p-1)}(1)+l_{p}^{1} y=0, \\
& \ell_{2, n+j} y \equiv y^{(2 j-2)}(0)-y^{(2 j-2}(1)=0, \quad j=1,2, \ldots, n .
\end{aligned}
$$

Let $L_{2, k} \equiv L_{2, k, p}$ be the operator of the problem (42)-(45) and

$$
\begin{gathered}
L_{2, k} y \equiv(-1)^{n} y^{(2 n)}(x)+z_{k}^{2 n} y(x), \\
y \in D\left(L_{2, k}\right), D\left(L_{2, k}\right) \equiv\left\{y \in W^{2 n}(0,1): l_{2, j} y=0, j=1,2, \ldots, 2 n\right\} .
\end{gathered}
$$

Theorem 5. Suppose that the Assumption $P_{1}$ holds. Then for arbitrary numbers $b_{p, r, s} \in \mathbb{R}, s=$ $0,1, r=0,1, \ldots, m_{p}, p \in\{1,2, \ldots, n\}$, the following statements hold

1) the eigenvalues of the operators $L_{0, k}$ and $L_{2, k}$ coincide;

2) the system $V\left(L_{2, k}\right)$ is complete and minimal in the space $H_{0}$;

3) if in addition the Assumption $P_{2}$ holds, then the system $V\left(L_{2, k}\right)$ is the Riesz basis of the space $H_{0}$.

Proof. The proof of part 1 of the theorem can be made in the same way as in Theorem 2.

Let us define the elements of the system $V\left(L_{2, k}\right)$. A direct substitution gives that the function $v_{2 q}(x), q=0,1, \ldots$, satisfies the conditions (43)-(45). Therefore, the root function of the operator $L_{2, k}$ with respect to the eigenvalue $\lambda_{q, k}$ is defined by

$$
\begin{aligned}
& v_{2 q}\left(x, L_{2, k}\right)=v_{2 q}\left(x, L_{0, k}\right), \quad q=0,1, \ldots, \\
& v_{2 q-1}\left(x, L_{2, k}\right)=v_{2 q-1}(x)+c_{q, p} y_{2, p}\left(x, \rho_{q}\right), \\
& c_{q, p}=-l_{p}^{1}\left(v_{2 q-1}(x)\right)\left(l_{2, p} y_{2, p}\left(x, \rho_{q}\right)\right)^{-1}, \quad q=1,2, \ldots
\end{aligned}
$$

Consequently $L_{2, k} \in Q_{0}(I)$. If the Assumption $P_{2}$ holds, then from the inequality $\left|l_{p, b}^{1} v_{2 q-1}\right| \leq K_{9}\left(\rho_{q}\right)^{2 p-2}$ we obtain the inequality

$$
\left|l_{p}^{1}\left(v_{2 q-1}(x)\right)\left(l_{2, n+p} y_{2, p}\left(x, \rho_{0, q}\right)\right)^{-1}\right| \leq K_{10}<\infty .
$$

Taking Theorem 4 into account, we obtain the third statement of the theorem.

Therefore, the operator $L_{2}$ has the following system of root functions in the space $H_{1}$

$$
V\left(L_{2}\right) \equiv\left\{v_{q, k}\left(x, L_{2}\right) \equiv v_{q}\left(x, L_{2, k}\right) v_{k}: q=\overline{0, \infty}, k=\overline{1, \infty}\right\} .
$$

Remark 5.2. The operator $L_{2}$ has a system of root functions in the means of equalities

$$
\begin{aligned}
& L_{2} v_{2 q-1, k}\left(x, L_{2}\right)=\lambda_{q, k} v_{2 q-1, k}\left(x, L_{2}\right)+\xi_{q, p} v_{2 q, k}\left(x, L_{2}\right), \\
& \xi_{q, p}=-4 \sqrt{2}\left(\rho_{q}\right)^{2 n-1} c_{q, p}, \quad q, k=1,2, \ldots, \\
& L_{2} v_{2 q, k}\left(x, L_{2}\right)=\lambda_{q, k} v_{2 q, k}\left(x, L_{2}\right), \quad q=0,1, \ldots, k=1,2, \ldots
\end{aligned}
$$


Theorem 6. Suppose that the Assumption $P_{1}$ holds. Then, for arbitrary numbers $b_{p, r, 0} \in \mathbb{R}$, $r=0,1, \ldots, m_{p}, p \in\{1,2, \ldots, n\}$, the following statements hold

1) the eigenvalues of the operators $L_{0}$ and $L_{2}$ coincide;

2) the system $V\left(L_{2}\right)$ is complete and minimal in the space $H_{1}$;

3) if in addition the Assumption $P_{2}$ holds, then the system $V\left(L_{2}\right)$ forms the Riesz basis of the space $H_{1}$.

Proof. Taking Theorem 5 into account, it is possible to determine the elements of a system $W\left(L_{2, k}\right)$ which is biorthogonal to the system $V\left(L_{2, k}\right)$ in the space $H_{0}$.

Therefore, there exists $W\left(L_{2}\right) \equiv\left\{w_{q, k}\left(x, L_{2}\right) w_{k}: q=0,1, \ldots, k=1,2, \ldots\right\}$ which is the biorthogonal system of functions to the system $V\left(L_{2}\right)$ in the space $H_{1}$.

Thus the second statement of the theorem is proved.

Suppose that the Assumption $P_{2}$ holds. Taking the inequalities (46) into account, we obtain the estimate

$$
\left\|R\left(L_{2}\right) ;\left[H_{1}\right]\right\| \leq K_{11}<\infty .
$$

From the Bari Theorem (see [9]) we obtain the statement: the system $V_{1}$ forms the Riesz basis in $H_{1}$.

5.2. Consider the spectral problem

$$
\begin{aligned}
& (-1)^{n} D_{x}^{2 n} w(x)+A^{2 n} w(x)=\lambda w(x), \\
& \ell_{j} w \equiv D_{x}^{2 j-1} w(0)-D_{x}^{2 j-1} w(1)+l_{j}^{1} w=0, \\
& \ell_{n+j} w \equiv D_{x}^{2 j-2} w(0)-D_{x}^{2 j-2} w(1)=0,
\end{aligned}
$$

where

$$
\ell_{j}^{1} w \equiv \sum_{r=0}^{m_{j}}\left(b_{j, r, 0} D_{x}^{r} w(0)+b_{j, r, 1} D_{x}^{r} w(1)\right), \quad j=1,2, \ldots, n .
$$

Let $L_{3}$ be the operator of the problem (50)-(53) and

$$
L_{3} u \equiv(-1)^{n} D_{x}^{2 n} u+A^{2 n} u, \quad u \in D\left(L_{3}\right), D\left(L_{3}\right) \equiv\left\{u \in H_{2}: l_{j} u=0, j=1,2, \ldots, 2 n\right\} .
$$

We find the solution of the spectral problem (50)-(53) as the product $w(x)=y(x) v_{k}$, $v_{k} \in V(A), k=1,2, \ldots$

To determine the unknown function $y \in W^{2 n}(0,1)$ we obtain the spectral problem

$$
\begin{aligned}
& (-1)^{n} y^{(2 n)}+z_{k}^{2 n} y=\lambda y, \quad \lambda \in \mathbb{C}, \\
& \ell_{j} y \equiv y^{(2 j-1)}(0)+y^{(2 j-1)}(1)+l_{j}^{1} y=0, \\
& \ell_{n+j} y \equiv y^{(2 j-2)}(0)-y^{(2 j-2)}(1)=0, \quad j=1,2, \ldots, n .
\end{aligned}
$$

Let $L_{3, k}$ be the operator of the problem (54)-(56);

$$
\begin{gathered}
L_{3, k} y(x) \equiv(-1)^{n} y^{(2 n)}(x)+z_{k}^{2 n} ; \\
y \in D\left(L_{3, k}\right) ; D\left(L_{3, k}\right) \equiv\left\{y \in W^{2 n}(0,1): l_{j} y=0, j=1,2, \ldots, 2 n\right\} ;
\end{gathered}
$$

let $V\left(L_{3, k}\right)$ be the system of root functions of the operator $L_{3, k}$.

We can prove that

$$
v_{2 q}(x) \in D\left(L_{3, k}\right), L_{3, k} v_{2 q}(x)=\lambda_{q, k} v_{2 q}(x), \quad q=0,1, \ldots
$$


Therefore,

$$
v_{2 q}\left(x, L_{3, k}\right) \equiv v_{2 q}(x), \quad q=0,1, \ldots
$$

The root functions of the operator $L_{3, k}$ are determined by the equalities

$$
v_{2 q-1}\left(x, L_{3, k}\right) \equiv v_{2 q-1}(x)+\sum_{p=1}^{n} c_{1, q, p} y_{2, p}\left(x, \rho_{q}\right), \quad q=1,2, \ldots
$$

Substituting the expression (58) into the boundary conditions (55), (56), we obtain

$$
c_{1, q, p}=-\sqrt{2} \sum_{r=0}^{m_{p}}(-1)^{r-2 p+1} b_{p, r, 0}\left(\rho_{q}\right)^{2+r-2 p}, \quad p=1,2, \ldots, n, q=1,2, \ldots
$$

Thus, the operator $L_{3, k}$ has the system of root functions (57)-(59) in the means of equalities

$$
\begin{aligned}
& L_{3, k} v_{2 q-1}\left(x, L_{3, k}\right)=\lambda_{q, k} v_{2 q-1}\left(x, L_{3, k}\right)+\xi_{q}^{0} v_{2 q}\left(x, L_{3, k}\right), \\
& \xi_{q}^{0}=2 \sqrt{2} n\left(\rho_{q}\right)^{2 n-1} \sum_{p=1}^{n} c_{p, q}, \quad q=1,2, \ldots, \\
& L_{3, k} v_{2 k}\left(x, L_{3, k}\right)=\lambda_{q, k} v_{2 k}\left(x, L_{3, k}\right), \quad q=0,1, \ldots
\end{aligned}
$$

Let $R\left(L_{3, k}\right)$ be the operator which acts as $V\left(L_{0, k}\right) \rightarrow V\left(L_{3, k}\right)$. From the formulas (37), (58), we obtain the relation

$$
R\left(L_{3, k}\right)=\prod_{p=1}^{n} R\left(L_{2, k, p}\right)=E+\sum_{p=1}^{n} S\left(L_{2, k, p}\right) .
$$

Therefore, we have the inclusion $R\left(L_{3, k}\right) \in G_{0}\left(L_{0, k}\right) \subset Q_{0}(I)$. Thus, we obtain the following statement.

Lemma 5.1. Suppose that the Assumption $P_{1}$ holds. Then, for the any fixed $b_{p, r, 0} \in \mathbb{R}, r=$ $0,1, \ldots, m_{p}, p=1,2, \ldots, n$, the system $V\left(L_{3, k}\right)$ is complete and minimal in the space $H_{0}$.

Consider the system $V_{2}$ of functions

$$
\begin{aligned}
& v_{0,2}(x) \equiv v_{0}(x), v_{2 q, 2}(x) \equiv v_{2 q}(x,), \quad q=1,2, \ldots, \\
& \left.v_{2 q-1,2}(x) \equiv\left(1+\tau_{2}\right)(1-2 x)\right) v_{2 q-1}(x), \\
& \tau_{2} \equiv W^{n}\left(W^{n-1}\right)^{-1} c_{b}, c_{b} \equiv \sum_{p=1}^{n} b_{p, 2 p-1,0} .
\end{aligned}
$$

Let $R_{2}=E+S_{2}$ be the operator which acts as $V\left(L_{0, k}\right) \rightarrow V_{2}$.

Using that $S_{2}: H_{0,1} \longrightarrow 0, S_{2}: H_{0,0} \longrightarrow H_{0}$, we obtain that $R_{2} \in Q_{0}(I)$.

Lemma 5.2. Suppose that the Assumptions $P_{1}, P_{2}$ hold. Then the system $V_{2}$ forms the Riesz basis in the space $H_{0}$.

The proof is carried out analogously in Lemma 3.1.

Remark 5.3. Suppose that the Assumptions $P_{1}, P_{2}$ hold. Then the following relations hold

$$
\left.v_{2 q-1}\left(x, L_{3, k}\right)=v_{2 q-1,2}(x)+\sum_{j=2}^{n} c_{j, q}^{1} y_{j}\left(x, \rho_{q}\right)+\left(\rho_{q}\right)^{-1} c_{q}^{2}(1-2 x)\right) v_{2 q-1}(x),
$$

where

$$
\left|c_{j, q}^{1}\right| \leq K_{12},\left|c_{q}^{2}\right| \leq K_{12}<\infty, \quad q=0,1, \ldots .
$$

Therefore, the systems $V\left(L_{3, k}\right)$ and $V_{2}$ are squarely close in the space $H_{0}$. 
Lemma 5.3. Suppose that the Assumptions $P_{1}, P_{2}$ hold. Then, for any fixed $b_{p, r, 0} \in \mathbb{R}, r=$ $0,1, \ldots, m_{p}, p=1,2, \ldots, n$, the system $V\left(L_{3, k}\right)$ forms the Riesz basis in the space $H_{0}$.

The statement follows from Lemma 5.1, Lemma 5.2, Remark 5.4 and the Bari Theorem (see [9]).

Theorem 7. Suppose that the Assumption $P_{1}$ holds. Then, for any $b_{p, r, 0} \in \mathbb{R}, r=0,1, \ldots, m_{p}$, $r, p=1,2, \ldots, n$, the following assertions are true

1) the eigenvalues of the operators $L_{0, k}$ and $L_{3, k}$ coincide;

2) the system $V\left(L_{3, k}\right)$ is complete and minimal in the space $H_{0}$;

3) if in addition the Assumption $P_{2}$ holds, then the system $V\left(L_{3, k}\right)$ forms the Riesz basis of the space $H_{0}$.

Proof. The proof of part 1 of the theorem follows from Theorem 3, the second statement follows from Lemma 5.1 and the third statement follows from Lemma 5.3.

Let

$$
V\left(L_{3}\right) \equiv\left\{v_{q, k}\left(x, L_{3}\right) \equiv v_{q}\left(x, L_{3, k}\right) v_{k} \in W_{1}: q=\overline{0, \infty}, k=\overline{1, \infty}\right\}
$$

forms the system of the root functions of the operator $L_{3}$. Let

$$
W\left(L_{3, k}\right) \equiv\left\{w_{q, k}\left(x, L_{3}\right) \in H_{1}: q=\overline{0, \infty}\right\}
$$

be the biorthogonal system of functions to the system $V\left(L_{3, k}\right)$ in the space $H_{0}$. Let

$$
W\left(L_{3}\right) \equiv\left\{w_{q, k}\left(x, L_{3}\right) \equiv w_{q}\left(x, L_{3, k}\right) w_{k} \in H_{1}: q=\overline{0, \infty}, k=\overline{1, \infty}\right\},
$$

be the biorthogonal system of functions to the system $V\left(L_{3}\right)$ in the space $W_{1}$ and $R\left(L_{3}\right)$ be the operator which acts as $V\left(L_{0}\right) \rightarrow V\left(L_{3}\right)$.

Theorem 8. Suppose that the Assumption $P_{1}$ holds. Then, for all numbers $b_{p, r, 0} \in \mathbb{R}$, $r=0,1, \ldots, m_{p}, p=1,2, \ldots, n$, the following assertions are true

1) the eigenvalues of the operators $L_{0}$ and $L_{3}$ coincide;

2) the system $V\left(L_{3}\right)$ is complete and minimal in the space $H_{1}$;

3) if in addition the Assumption $P_{2}$ holds, then the system $V\left(L_{3}\right)$ forms the Riesz basis of the space $H_{1}$.

Proof. The proof of part 1 and 2 of the theorem follows from Theorem 7. Taking the relations (60), (61) into account we obtain the equality

$$
R\left(L_{3}\right) \equiv \prod_{j=1}^{n} R\left(L_{2, j}\right) \equiv \prod_{j=1}^{n}\left(E+S\left(L_{2, j}\right)\right)=E+\sum_{j=1}^{n} S\left(L_{2, j}\right) .
$$

Let Assumptions $P_{1}$ and $P_{2}$ hold. Then from the equality (62) and the assertion 3 of Theorem 7 we obtain

$$
R\left(L_{3}\right) \in\left[H_{1}\right], R\left(L_{3}\right)^{-1}=E-S\left(L_{3}\right) \in\left[H_{1}\right] .
$$

Therefore, the system $V\left(L_{3}\right)$ forms the Riesz basis of the space $H_{1}$.

Remark 5.4. The operator $L_{3}$ has the system of root functions in the means of the equalities

$$
\begin{aligned}
& L_{3} v_{2 q-1, k}\left(x, L_{3}\right)=\lambda_{q, k} v_{2 q-1, k}\left(x, L_{3}\right)+\xi_{q, k}^{0} v_{2 q, k}\left(x, L_{3}\right), \\
& \xi_{q, k}^{0}=2 \sqrt{2} \tau_{2} n\left(\rho_{q}\right)^{2 n-1} \sum_{p=1}^{n} c_{p, q}, \quad q=1,2, \ldots \\
& L_{3} v_{2 q, k}\left(x, L_{3}\right)=\lambda_{q, k} v_{2 q-1, k}\left(x, L_{3}\right), \quad q=0,1, \ldots
\end{aligned}
$$


We consider the system of functions

$$
V_{3} \equiv\left\{v_{q, k, 3}(x) \in H_{1}: v_{q, k, 3}(x) \equiv v_{q, 1}(x) v_{k}, q=\overline{0, \infty}, k=\overline{1, \infty}\right\} .
$$

Remark 5.5. The systems $V\left(L_{3}\right)$ and $V_{3}$ are squarely close in the space $H_{1}$.

6 THE SPECTRAL BOUNDARY VALUE PROBLEM FOR A DIFFERENTIAL-OPERATOR EQUATION WITH INVOLUTION

Consider the spectral problem

$$
\begin{aligned}
& (-1)^{n} D_{x}^{2 n} u+A^{2 n} u+\sum_{s=1}^{n} a_{s}\left(D_{x}^{2 s-1} u(x)+D_{x}^{2 s-1} u(1-x)\right)=\lambda u, \\
& \ell_{j} u \equiv D_{x}^{2 j-1} u(0)-D_{x}^{2 j-1} u(1)+l_{j}^{1} u=0, \\
& \ell_{n+j} u \equiv D_{x}^{2 j-2} u(0)-D_{x}^{2 j-2} u(1)=0,
\end{aligned}
$$

with

$$
\ell_{j}^{1} u \equiv \sum_{r=0}^{m_{j}}\left(b_{j, r, 0} D_{x}^{r} u(0)+b_{j, r, 1} D_{x}^{r} u(1)\right), \quad j=1,2, \ldots, n .
$$

Let $L$ be the operator of the problem (63)-(66) and

$$
\begin{gathered}
L u \equiv(-1)^{n} D_{x}^{2 n} u+A^{2 n} u+\sum_{s=1}^{n} a_{s}\left(D_{x}^{2 s-1} u(x)+D_{x}^{2 s-1} u(1-x)\right) ; \\
u \in D(L), \quad D(L) \equiv\left\{u \in H_{2}: l_{j} u=0, j=1,2, \ldots, 2 n\right\} .
\end{gathered}
$$

We can prove that

$$
\begin{aligned}
& L v_{2 q-1, k}\left(x, L_{3}\right)=\lambda_{q, k} v_{2 q-1, k}\left(x, L_{3}\right)+\xi_{q}^{1} v_{2 q, k}\left(x, L_{3}\right), \quad q=1,2, \ldots, \\
& \xi_{q, k}^{1}=\xi_{q, k}^{0}+2 \sqrt{2} \tau_{2} \sum_{j=1}^{n}(-1)^{j-1} a_{j}\left(\rho_{q}\right)^{2 j-1}(-4 j+2), \quad q=1,2, \ldots, \\
& L v_{2 q, k}\left(x, L_{3}\right)=\lambda_{q, k} v_{2 q-1, k}\left(x, L_{3}\right), \quad q=0,1, \ldots
\end{aligned}
$$

Consequently, $V(L) \equiv V\left(L_{3}\right)$ and the following theorem is true.

Theorem 9. Suppose that the Assumption $P_{1}$ holds. Then for the any numbers $b_{p, r, 0}, a_{j} \in$ $\mathbb{R}, r=0,1, \ldots, m_{p}, j, p=1,2, \ldots, n$ we have 1) the eigenvalues of the operators $L_{0}$ and $L$ coincide;

2) the system $V(L)$ of the root functions of the operator $L$ is complete and minimal in the space $H_{1}$.

3) if in addition the Assumption $P_{2}$ holds, then the system $V(L)$ forms the Riesz basis in the space $H_{1}$.

Let

$$
f=\sum_{k=1}^{\infty} \sum_{q=0}^{\infty} f_{q, k} v_{q, k}(x, L), \quad f_{q, k}=\left(f, w_{q, k}(x, L) ; H_{1}\right) .
$$

Remark 6.1. From the definition of the Riesz basis of Hilbert space and the third statement of Theorem 9 for any $f \in H_{1}$ we obtain the relation

$$
K_{13}\left\|f ; H_{1}\right\|^{2} \leq \sum_{k=1}^{\infty} \sum_{q=0}^{\infty}\left|f_{q, k}\right|^{2} \leq K_{14}\left\|f ; H_{1}\right\|^{2}
$$


7 THE BOUNDARY VALUE PROBLEM WITH HOMOGENEOUS CONDITIONS WITH INVOLUTION

We consider the following boundary problem

$$
\begin{aligned}
& (-1)^{n} D_{x}^{2 n} w+A^{2 n} w+\sum_{s=1}^{n} a_{s}\left(D_{x}^{2 s-1} w(x)+D_{x}^{2 s-1} w(1-x)\right)=f, \\
& \ell_{j} w \equiv D_{x}^{2 j-1} w(0)-D_{x}^{2 j-1} w(1)+l_{j}^{1} w=0, \\
& \ell_{n+j} w \equiv D_{x}^{2 j-2} w(0)-D_{x}^{2 j-2} w(1)=0,
\end{aligned}
$$

where

$$
\ell_{j}^{1} w \equiv \sum_{r=0}^{m_{j}}\left(b_{j, r, 0} D_{x}^{r} w(0)+b_{j, r, 1} D_{x}^{r} w(1)\right), \quad j=1,2, \ldots, n .
$$

Let $W(L)$ be a biorthogonal system of functions from $V(L)$ in the space $H_{1}$.

Theorem 10. Suppose that the Assumption $P_{1}$ holds. Then for arbitrary numbers $b_{p, r, 0}, a_{j} \in \mathbb{R}$, $r=0,1, \ldots, m_{p}, j, p \in\{1,2, \ldots, n\}$, and function $f \in H_{1}$ there exists a unique solution of the problem (68)-(71).

Proof. The solution of the problem (68)-(71) can be determined by the relation

$$
w=\sum_{k=1}^{\infty} \sum_{q=0}^{\infty} w_{q, k} v_{q, k}(x, L) .
$$

Substituting the relations (67), (72) into the equation (68) we obtain

$$
w_{2 q-1, k}=\lambda_{q, k}^{-1} f_{2 q-1, k}, w_{2 q, k}=\lambda_{q, k}^{-1} f_{2 q, k}-\lambda_{q, k}^{-2} \xi_{q, k}^{1} f_{2 q-1, k}, \quad q, k=1,2, \ldots
$$

Therefore,

$$
\begin{aligned}
& \left|w_{2 q-1, k}\right|^{2} \leq K_{15}\left|f_{2 q-1, k}\right|^{2}, \\
& \left|w_{2 q, k}\right|^{2} \leq K_{16}\left(\left|f_{2 q-1, k}\right|^{2}+\left|f_{2 q, k}\right|^{2}\right), \quad q, k=1,2, \ldots
\end{aligned}
$$

Taking the assumption $f \in H_{1}$ and the inequalities (67) into account we obtain that $\left\|w ; H_{1}\right\| \leq K_{17}\left\|f ; H_{1}\right\|, w \in H_{1}$.

Consider the function $h_{1} \equiv A^{2 n} w$

$$
\begin{aligned}
h_{1} & =\sum_{k=1}^{\infty}\left(f_{0, k} v_{0, k}(x, L)+\sum_{q=1}^{\infty}\left(z_{k}^{2 n} \lambda_{q, k}^{-1} f_{2 q-1, k} v_{2 q-1, k}(x, L)\right.\right. \\
& \left.\left.+\left(z_{k}^{2 n} \lambda_{q, k}^{-1} f_{2 q, k}-\lambda_{q, k}^{-2} z_{k}^{2 n} \xi_{q, k}^{1} f_{2 q-1, k}\right) v_{2 q, k}(x, L)\right)\right) .
\end{aligned}
$$

Taking the assumption $f \in H_{1}$ and the inequalities (67) into account we obtain that $\left\|h_{1} ; H_{1}\right\| \leq K_{18}\left\|f ; H_{1}\right\|, h_{1} \in H_{1}$.

Consider the function $h_{2} \equiv(-1)^{n} D_{2}^{2 n} w$

$$
\begin{aligned}
h_{2} & =\sum_{k=1}^{\infty} \sum_{q=1}^{\infty}\left(\rho_{q}^{2 n} \lambda_{q, k}^{-1} f_{2 q-1, k} v_{2 q-1, k}(x, L)\right. \\
& \left.+\left(\rho_{q}^{2 n} \lambda_{q, k}^{-1} f_{2 q, k}-2 \rho_{q}^{2 n} \lambda_{q, k}^{-2} \xi_{q, k}^{1} f_{2 q-1, k}\right) v_{2 q, k}(x, L)\right) .
\end{aligned}
$$

From the equalities (76) we get $\left\|h_{2} ; H_{1}\right\| \leq K_{19}\left\|f ; H_{1}\right\|, h_{2} \in H_{1}$. 
Consider the function $h_{3}=\sum_{s=1}^{n} a_{s}\left(D_{x}^{2 s-1} w(x)-D_{x}^{2 s-1} w(1-x)\right)$

$$
h_{3}=2 \sum_{k=1}^{\infty} \sum_{q=1}^{\infty} \sum_{j=1}^{n} a_{j}(-1)^{j-1} \rho_{q}^{2 j-1}\left(\lambda_{q, k}^{-1} f_{2 q, k}-\lambda_{q, k}^{-2} \xi_{q, k}^{1} f_{2 q-1, k}\right) v_{2 q-1, k}(x, L) .
$$

Taking the assumption $f \in H_{1}$ and the equalities (77) into account we obtain that

$$
\left\|h_{3} ; H_{1}\right\| \leq K_{20}\left\|f ; H_{1}\right\| \text {. }
$$

From the definition of the space $H_{2}$, inequalities (75)-(77) and Cauchy's inequality we get

$$
\left\|w ; H_{2} \leq K_{21}\right\| f ; H_{1} \|<\infty, K_{21}=3\left(\max \left(K_{17}^{2}, K_{18}^{2}, K_{19}^{2}\right)\right)^{\frac{1}{2}} .
$$

Thus $w \in H_{2}$.

\section{REFERENCES}

[1] Ashyralyev A., Sarsenbi A.M. Well-posedness of an elliptic equations with an involution. Electr. J. Diff. Eq. 2015, 284, 1-8.

[2] Baranetskij Ya.O., Yarka U.B., Fedushko S.A. Abstract perturbations of the Dirichlet differential operator. Spectral properties. Scientific Bull. Uzhg. Univ. Ser. Mat. Inf. 2012, 3 (1), 12-16. (in Ukrainian)

[3] Baranetskij Ya., Basha A. Nonlocal multipoint problem for differential-operator equations of order $2 n$. J. Math. Sci. 2016, 217 (2), 176-186. doi:10.1007/s10958-016-2965-0

[4] Baranetskij Ya.O., Kalenyuk P.I., Kolyasa L.I. Boundary-value problem for abstract differential equations with operator involution. Bukov. Math. J. 2016, 4 (3-4), 22-29.

[5] Baranetskij Ya., Kolyasa L. Boundary-value problem for abstract second-order differential equation with involution. Visn. Lviv Polytech. National Univ. Ser. Phys. Math. Sci. 2017, 871, 20-27.

[6] Baranetskij Ya. O., Kalenyuk P.I., Kolyasa L.I., Kopach M.I. The nonlocal problem for the differentialoperator equation of the even order with the involution. Carpathian Math. Publ. 2017, 9 (2), 109-119. doi: 10.15330/cmp.9.2.109-119

[7] Burlutskaya M.Sh., Khromov A.P. Initial-boundary value problems for first-order hyperbolic equations with involution. Dokl. Math. 2011, 84 (3), 783-786. doi:10.1134/S1064562411070088 (translation of Dokl. Akad. Nauk 2011, 441 (2), 156-159. (in Russian))

[8] Cabada A., Tojo F.A.F. Existence results for a linear equation with reflection, non-constant coefficient and periodic boundary conditions. J. Math. Anal. Appl. 2014, 412 (1), 529-546. doi: 10.1016/j.jmaa.2013.10.067

[9] Gokhberg I. Ts., Krein M.G. Introduction to the Theory of Linear Non Self-Adjoint Operators. Nauka, Moscow, 1965. (in Russian)

[10] Gupta C.P. Two-point boundary value problems involving reflection of the argument. Int. J. Math. Math. Sci. 1987, 10 (2), 361-371. doi:10.1155/S0161171287000425

[11] Kirane M., Al-Salti N. Inverse problems for a nonlocal wave equation with an involution perturbation. J. Nonlinear Sci. Appl. 2016, 9, 1243-1251.

[12] Kritskov L.V., Sarsenbi A.M. Spectral properties of a nonlocal problem for the differential equation with involution. Differ. Equ. 2015, 51 (8), 984-990. doi:10.1134/S0012266115080029

[13] Kurdyumov V.P. On Riescz bases of eigenfunction of 2-nd order differential operator with involution and integral boundary conditions. Izv. Saratov Univ. (N.S.), Ser. Math. Mech. Inform. 2015, 15 (4), 392-405. doi:10.18500/1816-9791-2015-15-4-392-405 (in Russian).

[14] Moiseev E.I., Ambartsumyan V.E. On the Basis Property of the Eigenfunctions of the Frankl Problem with Nonlocal Evenness and Oddness Conditions of the Second Kind. Dokl. Math. 2010, 432 (4), 451-455. doi: 10.1134/S1064562410030257 (in Russian)

[15] Naimark M.A. Linear differential operators. Frederick Ungar Publ. Co., New York, 1967. 
[16] O'Regan D. Existence results for differential equations with reflection of the argument. J. Aust. Math. Soc. 1994, 57 (2), 237-260. doi:10.1017/S1446788700037538

[17] Sadybekov M.A., Sarsenbi A.M. Mixed problem for a differential equation with involution under boundary conditions of general form. AIP Conf. Proc. 2012, 1470. doi:10.1063/1.4747681

[18] Sadybekov M.A., Turmetov B.K. On analogues of periodic boundary value problems for the Laplace operator in a ball. Eurasian Math J. 2012, 3 (1), 143-146.

Received 04.02.2018

Revised 03.05.2018

Баранецький Я.О., Аемків I.І., Івасюк І.Я., Копач М.І. Нелокальна задача для диферениіальнооператорного рівняння порядку 2 з необмеженими операторними коефічієнтами з інволюиією // Карпатські матем. публ. - 2018. — Т.10, №1. — С. 14-30.

Вивчається нелокальна крайова задача для диференціально-операторного рівняння парного порядку, який містить оператор іноволюції. Аосліджується задача з періодичними крайовими умовами для диференціального рівняння, коефіцієнти якого $є$ несамоспрженими операторами. Встановлено, що оператор задачі має два інваріантні підпростори, породжені оператором інволюції та дві підсистеми системи власних функцій, які є базисами Рісса в кожному з підпросторів. Аля диференціально-операторного рівняння парного порядку вивчається задача з несамоспряженими крайовими умовами, які $€$ збуреннями періодичних умов. Вивчено випадки, коли збурені умови $є$ регулярними, але не сильно регулярними за Біркгофом та нерегулярними за Біркгофом. Визначено власні значення і елементи системи кореневих функцій $V$ оператора задачі, яка $є$ повною та містить нескінченне число приєднаних функцій. Отримано достатні умови, при яких система $V \in$ базисом Рісса. Визначено умови існування та єАиності розв' язку задачі з однорідними крайовими умовами, який побудовано у вигляді ряду за системою $V$.

Ключові слова і фрази: оператор інволюції, диференціально-операторне рівняння, власні функціі, базис Рісса. 